\title{
Chronic progressive external ophthalmoplegia vs. myasthenia gravis - case report and review of literature
}

\author{
Alexandru Dima', Alexandra Oprisan ${ }^{1,2}$, Alexandra E. Bastian ${ }^{3,4}$ \\ ${ }^{1}$ Department of Neurology, "Colentina" Clinical Hospital, Bucharest, Romania \\ 2Department of Neurology, "Carol Davila" University of Medicine and Pharmacy, Bucharest, Romania \\ ${ }^{3}$ Department of Pathology, Faculty of Dental Medicine, "Carol Davila" University of Medicine and Pharmacy, \\ Bucharest, Romania \\ ${ }^{4}$ Department of Pathology, "Colentina” Clinical Hospital, Bucharest, Romania
}

\begin{abstract}
The mitochondrial myopathy consists of a heterogeneous group of conditions characterized by primary disfunction of mitochondrial respiratory chain causing muscle disease. Chronic progressive external ophthalmoplegia is a frequent mitochondrial disorder that shares clinical, enzymatic and genetic features with other mitochondrial diseases. We present a case of a patient who was initially diagnosed with myasthenia gravis, did not respond to specific therapy and was subsequently diagnosed by clinical, biochemical and histopathological criteria with a mitochondrial myopathy. A 37 years old woman, suspected and treated for myasthenia gravis presented with ptosis, marked fatigue, muscle weakness and myalgia, symptoms that progressively got worse despite the anticholinesterase therapy. Knowledge of this entity allows us to avoid unnecessary treatment and deferral of the real diagnosis.
\end{abstract}

Keywords: chronic progressive external ophthalmoplegia, mitochondrial myopathy, ragged-red fibers, ragged-blue fibers, myasthenia gravis

\section{INTRODUCTION}

Mitochondrial myopathies (MM) consists of a wide, heterogeneous group of conditions characterized by primary dysfunction of the mitochondrial respiratory chain and causing a large range of clinical expressions with poor genotype-phenotype correlation. The majority of systems relying on aerobic metabolism are affected and involvement of the skeletal muscles and nervous system is common. Mitochondrial disorders are the most common form of inherited metabolic diseases with an incidence of 1 in 4,000 (1), while chronic progressive external ophthalmoplegia (CPEO) is the most common mitochondrial disease. According to the type of inheritance, age at onset and biochemical and genetic defects, mitochondrial myopathies may have various clinical presentation. There are some well-defined mitochondrial entities but there is often a clinical overlap between different patterns and the diagnosis requires certain biochemical, genetic and histopathologic criteria. CPEO is usually sporadic although familial forms have been described in one third of the reported cases with different patterns of inheritance: maternal, autosomal recessive and autosomal dominant (2). It is characterized by the development of a slowly progressive paresis of extraocular muscles along with bilateral ptosis in the fourth decade of life (3) although it can appear at any age. The upward gaze (4) is especially impaired but despite the fact that some patients present disconjugate gaze, diplopia is unusual or 
transitory. The patients may note muscle weakness and myalgia. The differential diagnosis of CPEO includes myasthenia gravis, thyroid ophthalmopathy, ocular myositis, oculopharyngeal muscular dystrophy and congenital fibrosis of the extraocular muscles (3).

We present the case of a 37 years old woman who was initially suspected and treated for myasthenia gravis, unresponsive to specific treatment.

\section{CASE PRESENTATION}

37 years old Caucasian women was admitted in Neurology Department with a one-year history of bilateral ptosis, inconstant diplopia, marked fatigue, global muscle weakness, exercise intolerance and diffuse myalgia. The symptoms affected her daily life activities, have no diurnal variation and progressively worsened. One year ago, when she first observed these changes, she presented for a neurological evaluation after which she was diagnosed with myasthenia gravis despite the absence of acetylcholine receptor antibodies and decrement on electromyography exam. The neostigmine test was performed without significant improvement, and pyridostigmine was initiated along with buthylscopolamine, pantoprazole and potassium supplements.

CT (computer tomography) chest scan was also performed but did not reveal any thymic abnormalities.

She had a medical history of myopia and tension-type headache, with no significant family medical history.

The physical examination was normal except for a low body mass index (BMI) of 17 (underweight), the patient mentioning a low body mass since her early twenties. Neurologic exam revealed bilateral ptosis, limited abduction and adduction with both eyes, limited vertical upward gaze, generalized muscular atrophy and generalized hypotony.

Blood tests showed creatine kinase $(\mathrm{CK})=$ 3,409 UI/1 (normal range 26-140 UI/1), CK-MB = 67.3 UI/1 (normal range 7-25 UI/1), values which decreased during hospitalization (CK on discharge $=264 \mathrm{UI} / 1$ ), slightly elevated lactate dehydrogenase $(\mathrm{LDH}=324 \mathrm{UI} / 1$ - normal range 135-220 UI/l), with normal comprehensive metabolic panel, liver function tests, electrolytes, thyroid function tests, immunologic (ANA antibody, anti dsDNA antibody, Anti RO, Anti La antibodies, rheumatoid factor) and infectious disease testing for HIV, hepatitis $\mathrm{B}, \mathrm{C}$ and treponema pallidum. A six-minute walk test revealed a normal resting lactic acid $=1.4$ $\mathrm{mmol} / \mathrm{l}$ and an elevated post-six-minute-walk lactic-acid $=4.1 \mathrm{mmol} / 1$ (normal result $<2 \mathrm{mmol} / 1$ ). The ammonia levels were normal both at rest and after six-minute walk.

ECG (electrocardiography), echocardiography and abdominal ultrasonography were normal. Brain MRI (magnetic resonance imaging) revealed chronic maxillary sinusitis, without brain or cranial nerves abnormalities. EMG (electromyography) and nerve conduction study showed velocities within normal range, normal motor units without spontaneous activity, no decrement or increment after repetitive stimulation, although myopathic findings were present in all examined muscles (tibialis anterior, vastus lateralis, deltoid muscles). The patient also underwent ophthalmological examination and electroretinogram which confirmed the diagnosis of myopia with no other pathologic findings.

A muscle biopsy was performed on the left deltoid muscle for light microscopy and biochemical

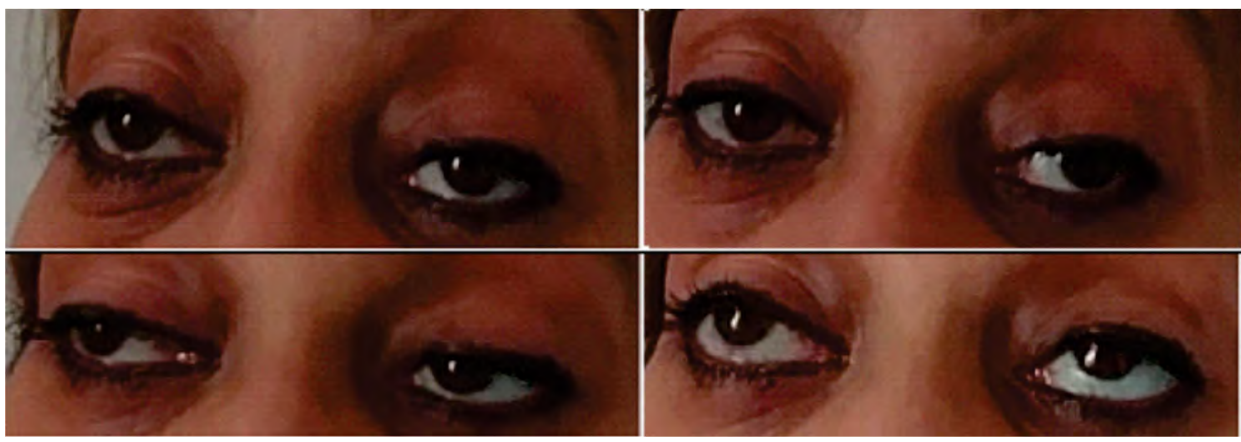

FIGURE 1. Bilateral ptosis, limited abduction and adduction with both eyes, limited vertical upward gaze 
evaluation which showed several abnormalities including moderate and severe atrophic muscle fibers. Histologic examination on H\&E (hematoxylin $\&$ eosin) stain was consistent with myopathy showing scattered degenerating muscle fibers. On Gomori trichrome stain scattered "ragged red fibers" were noted and "ragged blue fibers" on succinate dehydrogenase stain. Cytochrome oxidase $\mathrm{C}$ reaction revealed COX-negative fibers. These findings support the diagnosis of mitochondrial myopathy.

The confirmed CPEO diagnosis or any other forms of mitochondrial myopathy requires genome sequencing which is not routinely available and may be performed in genetic centers.

Once the diagnosis is highly probable or confirmed, the treatment aims to reduce morbidity, improve quality of life and allows the physician to give genetic counseling. Having a correct diagnosis also prevents the patient being misdiagnosed and mistreated for other diseases, saving the patient from potential severe adverse reactions which would alter even more the quality of life, without significant clinical improvement. There is no disease modifying therapy available for mitochondrial myopathies and several agents, mostly nutritional supplements have been taken into consideration but without significant efficacy (6).

Our patient received coenzyme Q10 $600 \mathrm{mg}$ per day, L-carnitine $330 \mathrm{mg}$ daily, creatine $4.5 \mathrm{~g}$ daily, thiamine and pyridoxine. She is recommended mild to moderate aerobic exercises that appears to be beneficial in mitochondrial disorders being associated with increased peak work, oxidative capacity and mitochondrial volume (7). Aerobic exercise also prevents muscle deconditioning and decreases exercise intolerance (8). On follow-up evaluations at 6 months, respectively at one year after therapy initiation there was neither a significant improvement nor worsening of the clinical presentation. Taking into consideration that mitochondrial myopathies usually affect multiple organs and systems, the patient is periodically monitored by echocardiography, ECG and undergoes ophthalmological examination every year.

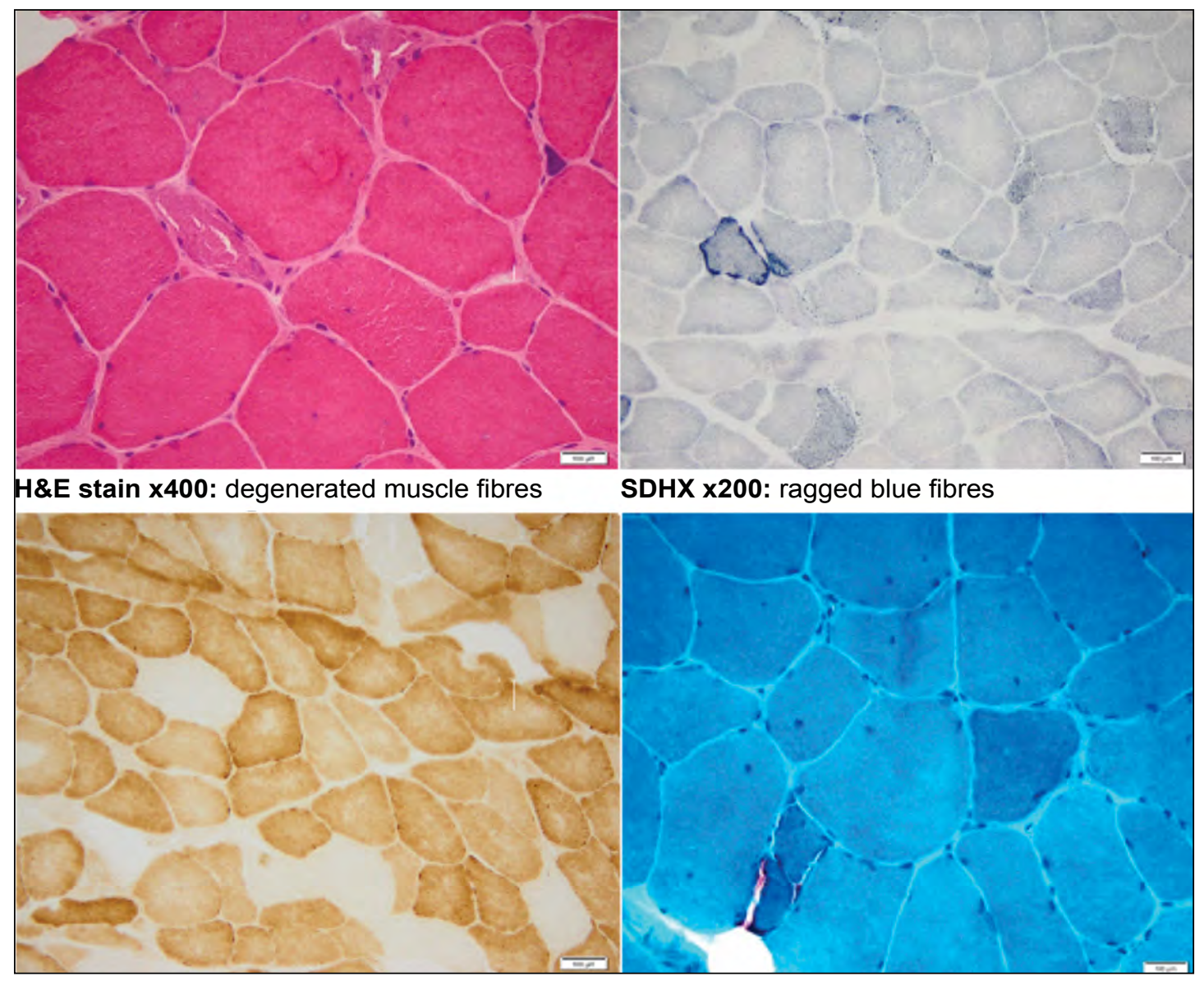




\section{DISCUSSIONS}

Progressive external ophthalmoplegia is a notable but nonspecific clinical sign that appears in a variety of pathological conditions such as myasthenia gravis, Guillain-Barré syndrome, thyroid associated orbitopathy, Refsum's disease, Bassen-Kornzweig disease (9), oculopharyngeal muscular dystrophy and congenital fibrosis of the extraocular muscles (3).

The prevalence of mitochondrial myopathies was estimated to be $0.025 \%$, but there is noticeable difficulty in diagnosis of the disease due to clinical and genetic heterogeneity (6).

Mitochondrial myopathies are characterized by morphological abnormalities of mitochondria, especially muscle mitochondria. Similar to myasthenia gravis patients may present with ptosis, diplopia, muscle weakness, exercise intolerance and fatigue that progressively worsens over time without proper treatment.

Patients with chronic progressive external ophthalmoplegia usually develop a slowly progressive paresis of extraocular muscles and bilateral ptosis in the fourth decade of life, although it can occur at any age (11). Patients with either CPEO or KSS usually develop proximal myopathy (3). Kearns-Sayre syndrome (KSS) is a combination of $\mathrm{CPEO}$ and pigmentary retinopathy with onset before age 20. Many other abnormalities have been described in association with Kearns-Sayre syndrome, like short stature, high CSF proteins $(<1$ g/l) (2), cerebral ataxia, anemia, diabetes, cardiac conduction defects, deafness, dementia, seizures and stroke-like episodes, therefore the need of periodic multidisciplinary evaluation of these patients. Kearns-Sayre syndrome is more severe than isolated CPEO, progressing to complete ophtalmoparesis and often the patients die by the fourth decade due to associated deficits (12).

Inheritance can be maternal, autosomal dominant, autosomal recessive, suggesting that both defects of mitochondrial and nuclear DNA may generate similar or identical phenotypes (3). Autosomal dominant and autosomal recessive forms of CPEO are linked to mutation of several nuclear DNA genes including POLG, C10orf2, RRM2B, SLC25A4, POLG2, DGUOK and SPG7 (13-17), while sporadic CPEO frequently originates from mutations affecting protein synthesis like large scale deletions or duplications (3). Genetic tests should be performed when available. They offer the base for genetic counseling which is an important concern particularly in young patients, like ours, who has three young daughters.

The diagnosis is based on patient history, clinical presentation, muscle biopsy and genetic testing with signs of active or inactive myopathy on electromyography (1).

Suggestive features are seen on microscopy, including deletion of "ragged red fibers" on Gomori trichrome stain and "ragged blue fibers" on SDH stain. COX deficient and SDH positive muscle fibers are sensitive and specific signs for the diagnosis of mitochondrial myopathy but cannot differentiate between the multiple forms of the disease (11).

There is no disease modifying treatment available, but several agents have been tried, mainly nutritional supplements, in an attempt to reduce morbidity and improve quality of life.

The patients have to undergo periodic cardiac, ophthalmologic and pulmonary evaluation along with neurologic monitoring, in order to detect possible complications.

The differential diagnosis of CPEO is mainly ocular myasthenia gravis which shares similar clinical presentation with ptosis, ophthalmoplegia and diplopia worsening after exercise.

\section{CONCLUSIONS}

Mitochondrial myopathies are one of the most frequent metabolic disorders and CPEO is one of the most frequent form of mitochondrial myopathy. Ocular involvement is a central sign of CPEO just like for ocular myasthenia gravis. In some cases may be difficult to differentiate between these two and diagnostic errors appear. If ocular myasthenia is suspected but the clinical presentation is atypical, the patient has normal acetylcholine receptor antibodies levels, no decrement on electromyography and does not respond to anticholinesterase treatment, one must also consider CPEO and a muscle biopsy should be performed. Genetic testing is particularly helpful to distinguish between various form of MM but also to offer a base for genetic counseling, which is of utmost importance in women at childbearing age.

\section{Acknowledgement}

Alexandra L. Oprisan and Alexandra E. Bastian equally contributed to this work. 


\section{REFERENCES}

1. Milone M, Wong LJ. Diagnosis of mitochondrial myopathies. Molecular Genetics and Metabolism Journal. 2013;110:35-41.

2. Caballero PE, Candela MS, Alvarez $\mathrm{Cl}$, Tejerina AA. Chronic progressive external ophthalmoplegia: A report of 6 cases and a review of the literature. Neurologist. 2007;13(1):33-6.

3. O'Ferrall E. Mitochondrial myopathies: Clinical features and diagnosis - https://www. uptodate.com/contents/mitochondrialmyopathies-clinical-features-and-diagnosis.

4. Hart PE, De Vivo DC, Schapira AH. Clinical features of the mitochondrial encephalomyopathies. In: Shapira AH, DiMauro S, editors. Mitochondrial Disorders in Neurology. Vol. 2. Woburn: Butterworth-Heineman; 2002. pp. 35-68.

5. Sharp LK, Haller RG. Metabolic and mitochondrial myopathies. Neurologic Clinics. 2014;32:777-99.

6. Kumari A, Goyal S, Malik V, Singh TB, Paramasivan VK, Kameswaran M. A rare case of dysphonia in mitochondrial myopathy. Int J Otorhinolaryngology Head Neck Surg. 2017;3:745.

7. Jeppesen TD, Schwartz M, Olsen DB et al. Aerobic training is safe and improves exercise capacity in patients with mitochondrial myopathy. Brain. 2006;129:3402.

8. Cejudo P, Bautista J, Montemayor T et al. Exercise training in mitochondrial myopathy: A randomized controlled trial. Muscle Nerve. 2005; 32:342.

9. Olson W, Tenn N, Engel WK, Walsh GO, Einaugler R:

Oculocraniosomatic neuromuscular disease with "ragged red fibers". Histochemical and ultrastructural changes in limb muscles of a group of patients with idiopathic progressive external ophtalmoplegia. Arch
Neurol. 1972;26:193-211.

10. Chinnery PF, Johnson MA, Wardell TM, Sigh-Kler R, Hayes C, Brown DT, Taylor RW, Bindoff LA, Turnbull DM. The epidemiology of pathogenic mitochondrial DNA mutations. Ann Neurol. 2000;48:188193.

11. DiMauro S. Pathogenesis and treatment of mitochondrial myopathies: Recent advances. Acta Myol. 2010;29:333-8.

12. Yamashita S, Nishino I, Nonaka I, Goto Y. Genotype and phenotype analyses in 136 patients with single large-scale mitochondrial DNA deletions. J Hum Genet. 2008;53:598.

13. Milone M, Massie R. Polymerase gamma 1 mutations: Clinical correlations. Neurologist. 2010;16:84.

14. Milone M, Benarroch EE, Wong LJ. POLG-related disorders: Defects of the nuclear and mitochondrial genome interaction. Neurology. 2011;77:1847.

15. Pitceathly RD, Smith $C$, Fratter $C$ et al. Adults with RRM2B-related mitochondrial disease have distinct clinical and molecular characteristics. Brain. 2012;135:3392.

16. Ronchi D, Garone C, Bordoni A et al. Next-generation sequencing reveals DGUOK mutations in adult patients with mitochondrial DNA multiple deletions. Brain. 2012;135:3404.

17. Pfeffer G, Gorman GS, Griffin H et al. Mutations in the SPG7 gene cause chronic progressive external ophthalmoplegia through disordered mitochondrial DNA maintenance. Brain. 2014;137:1323.

Conflict of interest: none declared Financial support: none declared 